\title{
Correção de hipotropia na posição primária do olhar mediante a tenectomia unilateral do músculo oblíquo superior próximo a inserção em estrabismo essencial
}

\author{
Correction of hypotropia in the primary position with unilateral superior oblique \\ tenectomy close to the scleral insertion
}

Simone Finzi (1)

Mauro Goldchmit ${ }^{(2)}$

Carlos Souza-Dias ${ }^{(3)}$

\section{RESUMO}

Objetivo: Os estrabismos horizontais essenciais estão freqüentemente associados a desvios verticais. A patogênese desse desvio vertical pode resultar da disfunção de músculos retos verticais, de músculos oblíquos ou da combinação de ambos. Na presença de hiperfunção do músculo oblíquo superior (OS), nota-se hipotropia (HoT) na posição primária do olhar (PPO). O presente estudo objetivou avaliar a magnitude da correção da HoT, na PPO, mediante a tenectomia unilateral do OS.

Pacientes $e$ Método: Foi realizado um estudo retrospectivo, 1977 a 1996, de 15 pacientes portadores de hiperfunção unilateral do OS e hipotropia na posição primária do olhar maior que $4^{\Delta}$, submetidos a tenectomia unilateral do OS, realizada na Santa Casa de São Paulo (12 pacientes), Universidade de Santo Amaro (2 pacientes) e na clínica particular de um dos autores (CSD, 1 paciente). A média de desvio pré-operatória era de $9^{\Delta}$. A hiperfunção média pré-operatória do músculo obliquo superior era 1,7 cruzes.

Resultados: A correção média da HoT obtida foi de $4,67^{\Delta} \pm 5,09^{\Delta}$ $\left(-5^{\Delta}\right.$ a $\left.15^{\Delta}\right),(H=6,032 ; p=0,014)$. A modificação média da hiperfunção do OS foi de $0,87 \pm 0,88$ cruzes ( 0 a 2 cruzes). De acordo com o desvio horizontal, ET e XT, não houve diferença estatisticamente significante na comparação entre os resultados obtidos na correção da HoT.

Comentários: Os resultados revelaram que para HoT até $15^{\Delta}$ na PPO, houve em média correção de $51,82 \%$ do seu valor pré-operatório. Para a amostra estudada, a técnica de tenectomia unilateral do OS mostrou-se eficaz na correção do desvio vertical na posição primária do olhar.

Palavras-chave: Estrabismo; Oblíquo superior; Tenectomia.

\section{INTRODUÇÃ̃O}

Os estrabismos horizontais essenciais estão freqüentemente associados a desvios verticais, dificultando a instalação da visão binocular. Nestes casos, a correção cirúrgica tem apenas finalidade estética ${ }^{9}$.

A causa desse desvio vertical pode ser a disfunção de músculos retos verticais, de músculos oblíquos, ou a combinação de ambos. Na presença da hiperfunção unilateral do músculo oblíquo superior (OS), pode observar-se hipotropia (HoT) na posição primária do olhar (PPO), que se acentua na lateroversão em que o olho com hiperfunção aduz (desvio vertical 


\section{ANÚNCIO}




\section{ANÚNCIO}


incomitante). A diferença na magnitude da HoT, nas diferentes posições do olhar no plano horizontal que, com base na fisiologia motora do músculo OS, define a indicação para seu debilitamento, corrigindo não somente a HoT na PPO, como também a incomitância às lateroversões ${ }^{9}$.

Diversas técnicas cirúrgicas foram descritas para o debilitamento cirúrgico do músculo OS 1, 3-5, 7, 8, 10, 11. VEMPALLI e LEE ${ }^{13}$ realizaram estudo para avaliar o resultado da tenectomia parcial posterior para a correção da HoT, em 14 pacientes. A correção média foi de $6,78^{\Delta}(\mathrm{p}<0,001)$. Em 8 dos 14 pacientes, houve redução da HoT maior que $50 \%$ de seu valor pré-operatório. Porém, na amostra desse estudo, as HoT incluídas possuíam etiologias diversas não considerando apenas os estrabismos essenciais. Apenas em 4 pacientes, a hiperfunção era secundária à hipofunção primária do OI. Entre os 14 pacientes, 10 haviam sido submetidos à cirurgia de estrabismo previamente.

No estudo realizado por BUCKLEY e FLYNN ${ }^{2}$, foram analisados os resultados da correção do desvio vertical por meio da técnica de tenectomia unilateral do OS (5 pacientes) e retrocesso unilateral do OS (4 pacientes). A média préoperatória do desvio vertical foi respectivamente $10,6^{\Delta} \mathrm{e}$ $10,7^{\Delta}$. Foi obtida maior correção pela tenectomia $\left(11^{\Delta}\right)$ do que pelo retrocesso $\left(6,2^{\Delta}\right)$.

Não há, que se saiba, trabalho publicado sobre a correção do desvio vertical em PPO por intermédio da tenectomia unilateral do OS junto à inserção escleral. Este estudo objetivou avaliar a magnitude da correção da HoT na PPO por esta técnica cirúrgica proposta por Souza-Dias em 1986, em pacientes com estrabismo essencial.

\section{MATERIAL E MÉTODO}

Avaliaram-se os resultados da HoT em 15 pacientes portadores de estrabismo essencial (10 pacientes com esotropia, 4 com exotropia e 1 com apenas desvio vertical na PPO) associado à hiperfunção unilateral do músculo oblíquo superior, através do debilitamento deste músculo pela técnica da tenectomia junto à inserção escleral, proposta por Souza-Dias em $1986^{10}$, e também realizada a correção do desvio horizontal no mesmo ato cirúrgico. Os pacientes eram provenientes da Secção de Motilidade Extrínseca Ocular da Disciplina de Oftalmologia da Santa Casa de São Paulo (12 pacientes), da Secção de Motilidade Extrínseca Ocular da Disciplina de Oftalmologia da Universidade Santo-Amaro (2 pacientes) e da clínica particular do Dr. Carlos Souza-Dias (1 paciente). As operações foram realizadas no período de 1977 a 1996.

Previamente ao exame da motilidade extrínseca ocular, todos os pacientes foram submetidos ao exame externo, medida da acuidade visual, refratometria e fundoscopia. Realizou-se a medida do ângulo de desvio por meio dos testes de prisma e "cover" ou Krimsky. Estabeleceu-se, por convenção que o sinal (-) corresponde a aumento da HoT. A função do OS foi avaliada pelo estudo das versões e quantificada em cruzes: 0: ausente; 0,5: discreta; 1 : leve; 2 : moderada; 3 : acentuada e 4 : severa.
Considerou-se haver HoT com indicação cirúrgica quando o desvio vertical era igual ou maior a $4^{\Delta}$. Definiu-se ambliopia como a diferença na medida da acuidade visual entre os olhos de no mínimo 2 linhas na tabela de optotipos de Snellen e observou-se, neste estudo, em 11 pacientes.

Os critérios para exclusão da amostra foram: intervenção cirúrgica de músculo (s) reto (s) vertical (is) ou transposição vertical de músculo (s) reto(s) horizontal (is) no mesmo ato operatório, existência de divergência vertical dissociada ou outra síndrome envolvendo o musculatura extrínseca ocular, como síndrome de Duane, síndrome de Möbius, fibrose generalizada de Brown, órbito-miopatia infiltrativa de BasedowGraves, etc.

Propôs-se, como critério de cura, desvio vertical menor que $4^{\Delta}$. O seguimento mínimo pós-operatório estabelecido foi de 1 mês; o seguimento médio, de 18,53 $\pm 18,37$ meses ( 1 a 72 meses). A idade média dos pacientes, na época da operação, era de 17,93 \pm 9,85 (7 a 35 anos).

Avaliou-se a correção da HoT separadamente, em pacientes com esotropia ou exotropia (Tabela 1). Para análise dos resultados, aplicou-se o teste de Kruskal Wallis para comparação dos desvios verticais pré e pós operatórios, e o teste de médias com amostras independentes, para a comparação dos valores da correção da HoT, entre os grupos de pacientes com eso e exotropia.

\section{RESULTADOS}

A correção média da HoT em PPO obtida foi de $4,67^{\wedge} \pm 5,09^{\wedge}$ $\left(-5^{\Delta}\right.$ a $\left.15^{\Delta}\right)$, o que corresponde à redução média de $51,82 \%$ do valor inicial da HoT. Houve correção integral da HOT em 7 pacientes (47\%), 5 portadores de ET, 1 de XT e 1 sem desvio horizontal na PPO. Observou-se aumento da HoT em 2 casos, ambos portadores de ET; em um paciente, o aumento foi de $5^{\Delta}$ e, no outro, de $2^{\Delta}$.

A redução média da hiperfunção do OS foi de $0,87 \pm 0,88$ cruzes ( 0 a 2) e a correção máxima, de $3+$. Houve subcorreção em 6 casos (40\%), a máxima, de $1+$. (Tabela 1). Não houve supercorreção em nenhum caso.

Utilizando o teste de Kruskal-Wallis com $\alpha$ de 5\% para a comparação da HoT pré e pós operatória, observa-se variação estatisticamente significante $(H=6,032, p=0,014)$. Em relação à hiperfunção, aplicou-se o mesmo teste e a variação encontrada também foi significativa ( $H=7,902, p=0,005$ ).

Compararam-se os resultados obtidos quanto à correção da HoT segundo o desvio horizontal na posição primária, ET e $\mathrm{XT}$. Utilizou-se o teste de médias para amostras independentes e verificou-se não haver diferença estatisticamente significante entre os dois grupos $(\mathrm{V}=24,86 \mathrm{e} \mathrm{p}=0,3617)$. (Tabela 2$)$.

\section{COMENTÁRIOS}

Não se encontra na literatura, outro trabalho que avalie a correção do desvio vertical em estrabismo essenciais na PPO, 
Correção de hipotropia na posição primária do olhar mediante a tenectomia unilateral do músculo oblíquo superior próximo a inserção em estrabismo essencial

ANÚNCIO 


\begin{tabular}{|c|c|c|c|c|c|c|c|}
\hline & DH & HoT pré & Desvio vertical pós & $\alpha$ & Hiperfunção pré & Hiperfunção pós & $\alpha$ \\
\hline 1 & ET45 & 15 & 0 & 15 & 1 & 0,5 & 0,5 \\
\hline 2 & ET35 & 10 & 10 & 0 & 1,5 & 0 & 1,5 \\
\hline 3 & ET30 & 7 & 12 & -5 & 1 & 0,5 & 0,5 \\
\hline 4 & ET65 & 4 & 0 & 4 & 1 & 1 & 0 \\
\hline 5 & ET30 & 5 & 0 & 5 & 2 & 0,5 & 1,5 \\
\hline 6 & ET25 & 7 & 0 & 7 & 1 & 1 & 0 \\
\hline 7 & ET75 & 10 & 10 & 0 & 3 & 2 & 1 \\
\hline 8 & ET85 & 8 & 10 & -2 & 1 & 1 & 0 \\
\hline 9 & ET27 & 6 & 2 & 4 & 2 & 2 & 0 \\
\hline 10 & ET45 & 10 & 0 & 10 & 2 & 0 & 2 \\
\hline 11 & XT45 & 12 & 4 & 8 & 1 & 0 & 1 \\
\hline 12 & XT50 & 5 & 0 & 5 & 1 & 1 & 0 \\
\hline 13 & XT40 & 10 & 6 & 4 & 3 & 2 & 1 \\
\hline 14 & XT40 & 15 & 10 & 5 & 2 & 1 & 1 \\
\hline 15 & $\mathrm{O}$ & 10 & 0 & 10 & 3 & 0 & 3 \\
\hline M & & 9 & 4 & 4,67 & 1,7 & 0,83 & 0,87 \\
\hline DP & & 3 & 5 & $5,090,80$ & 0,73 & 0,88 & \\
\hline \multicolumn{8}{|c|}{$\begin{array}{l}\text { Teste de Kruskal-Wallis ( } \alpha \text { de } 5 \% \text { ) para HoT: } \mathrm{H}=6,032 \quad p=0,014 \\
\text { Teste de Kruskal-Wallis ( } \alpha \text { de } 5 \% \text { ) para hiperfunção do OS: } \mathrm{H}=7,902 \quad \mathrm{p}=0,005\end{array}$} \\
\hline \multicolumn{8}{|c|}{$\begin{array}{l}\text { FONTE: Departamento de Oftalmologia da Santa Casa de São Paulo (12 pacientes), Departamento de Oftalmologia da Universidade Santo-Amaro (2 pacientes) e } \\
\text { clínica particular do Dr. Carlos Souza-Dias (1 paciente). DH: desvio horizontal na posição primária do olhar. HoT: hipotropia. } \alpha \text { : correção. M: média. DP: desvio padrão. } \\
\text { ET: esotropia. XT; exotropia. O desvio horizontal e o vertical foram quantificados em dioptrias prismáticas. A função muscular foi quantificada em cruzes (0: ausente; } \\
\text { 0,5: discreta 1: leve; 2: moderada; 3: acentuada; 4: severa). }\end{array}$} \\
\hline
\end{tabular}

mediante a tenectomia unilateral do músculo OS, o que evidencia a importância deste estudo.

A pequena magnitude da amostra prende-se ao fato da raridade dos casos em que se opera apenas um dos músculos OS com a intenção de corrigir a HoT. A técnica da tenectomia foi escolhida para este estudo, por ser uma das mais realizadas na prática dos serviços de onde os pacientes são provenientes, oferecendo, assim, amostra suficiente para análise estatística.

Os resultados relevaram que, para HoT até $15^{\Delta}$ na $\mathrm{PPO}$, houve em média correção de $4,67^{\Delta} \pm 5,09^{\Delta}$, correspondendo a $51,82 \%$ de seu valor pré-operatório.

Tabela 2. Média e Variância da correção da hipotropia segundo o desvio horizontal, $\mathbf{n}$ posição primária do olhar, eso ou exotropia, de 14 pacientes submetidos ao debilitamento por intermédio da técnica da tenectomia junto à inserção escleral, realizada no período de 1977 a 1996, em São Paulo.

$\begin{array}{lcc} & \text { Esotropia }(\mathbf{n = 1 0}) & \text { Exotropia }(\mathbf{n}=\mathbf{4}) \\ \text { Média } & 3,8 & 6,75 \\ \text { Variância } & 35,07 & 7,58 \\ \text { Teste de média para amostras independentes: } & \\ \text { V =24,86 } & \\ \mathrm{p}=0,317 & \\ \text { FONTE: Departamento de Oftalmologia da Santa Casa de Misericórdia de } \\ \text { São Paulo; Departamento de Oftalmologia da Faculdade de Medicina Santo- } \\ \text { Amaro e da clínica particular do Dr. Carlos Souza-Dias. }\end{array}$

Sete pacientes tiveram correção total da HoT, dos quais 5 pertenciam ao grupo de ET (71\%), enquanto somente 1 paciente possuía XT e o outro, apenas desvio vertical. Como em adução o oblíquo superior tem maior função depressora; esperava-se que houvesse maior correção da HoT nas ET, porém não se observou diferença estatisticamente significante entre os pacientes com ET e XT. Talvez uma das razões para o ocorrido fosse o tamanho da amostra, apenas 4 pacientes com XT.

Quanto à correção da hiperfunção do OS operado, notou-se que foi insatisfatória na maioria dos casos, pois constatou-se subcorreção em 6 casos (40\%) e não houve alteração da função em 5 casos (33\%); no entanto, entre estes 5 pacientes, houve correção total do desvio vertical em 3; portanto, a hiperfunção pós-operatória do músculo OS tem valor restrito na avaliação do sucesso da cirurgia destinada à correção da HoT.

Alguns estudos sugerem que a disfunção do OS não seja o único fator patogênico, indicando provável existência de fator desconhecido ${ }^{6,10}$. Nesse estudo, esse fato pode ser considerado pela fraca correlação que encontramos entre o valor da HoT pré-operatória com o grau de hiperfunção do músculo oblíquo superior, bem como a correção obtida. Obtivemos resposta paradoxal em 2 pacientes em que houve aumento da hipotropia pré-operatória após o debilitamento do OS, fato que não temos explicação. 
Destaca-se, ainda, que a correção da HoT no trabalho foi menor do que a correção descrita anteriormente, com a utilização de outras técnicas: tenectomia parcial posterior $\left(6,78^{\Delta}\right){ }^{12}$, tenotomia unilateral $\left(11^{\Delta}\right)^{2}$ e retrocesso unilateral $\left(6,25^{\Delta}\right)^{2}$, apesar da amostra estudada neste trabalho diferir dos demais, pois foram utilizados somente pacientes com estrabismo essencial.

A variabilidade de resultados por meio das diferentes técnicas pode decorrer do fato da linha de inserção do OS ser mais variável de todos os músculos extra-oculares, com largura média de $11 \mathrm{~mm}$, variando de 7 a $18 \mathrm{~mm}$.

A técnica da tenectomia unilateral do OS junto à inserção escleral mostrou-se eficaz na correção do desvio vertical, para HoT até $15^{\Delta}$, na amostra estudada. Novos que avaliem a correção cirúrgica do desvio vertical, mediante o debilitamento unilateral do OS com as demais técnicas, devem ser feitos para ficar demonstrada, assim, a eficácia desta técnica para correção da hipotropia em comparação com elas.

\section{SUMMARY}

Purpose: Horizontal strabismus is commonly associated with vertical deviations which can be a result of a dysfunction of the vertical rectus, oblique muscle or both. Overaction of the superior oblique muscle (SO) results in hypotropia (HoT) in primary position of gaze (PP). The aim of this study was to evaluate the correction of HoT in PP after unilateral tenectomy of the superior oblique muscle close to its scleral insertion.

Patients and Methods: We retrospectively studied, from 1977 until 1996, charts of 15 patients with SO overaction and hypotropia in PP greater than $4^{4}$ who underwent unilateral superior oblique tenectomy close to its insertion at Hospital Santa Casa de São Paulo (12 patients), University of Santo Amaro (2 patients) and private clinic of one of the authors (CSD, 1 patient). The mean preoperative deviation in PP was
$9^{4}$. The mean preoperative superior oblique overaction was 1.7 (measured by a cross system rating).

Results: The mean correction of the HoT was $4.67^{4} \pm 5.09^{4}$ $\left(-5^{\Delta}\right.$ to $\left.15^{4}\right),(H=6.032 ; p=0.014)$. The mean correction of $S O$ overaction was $0.87 \pm 0.88+(0-2)$. According to the horizontal deviation, ET and XT, there was no significant mdifference in the correction of the HoT.

Conclusion: For HoT of $15^{\Delta}$ or less we found a mean correction of $51.82 \%$ of its preoperative value. Unilateral tenectomy has shown to be an effective technique for the correction of vertical deviation in PP in our study.

Keywords: Strabismus; Oblique superior muscle; Tenectomy.

\section{REFERÊCIAS BIBLIOGRÁFICAS}

1. Berke RN. Tenotomy of the superior oblique for hypertropia. $\operatorname{Tr} \mathrm{Am}$ Ophthalmol Soc 1946;44:304-42.

2. Buckley EG, Flynn JT. Superior oblique recession versus tenotomy. A comparison of surgical results. J Ped Ophtalmology \& Strabismus 1983;20(3):112-7.

3. Caldeira JAF. Graduated recession of the superior oblique. Brit J Ophthalmol 1975;59:553-9.

4. Ciancia AO, Prieto-Díaz J. Retrocesso del oblicuo superior. Arch Oftalmol B Aires 1970;45:193-200.

5. Gobin $\mathrm{MH}$. Desinsertion of the superior oblique in "A" pattern strabismus. Documenta Ophthalmol 1977;44:193-202.

6. Goldchmit M. Eficácia da desinserção e da tenectomia bilaterais e simétricas dos músculos oblíquos superiores para a correção de anisotropia em "A", Tese, Universidade Federal de São Paulo, 1997.

7. Jampolsky A. Oblique muscle surgery of the A-V patterns. J Ped Ophtalmol 1965;2:31-6.

8. Prieto-Díaz J. Tenectomia parcial del obliquo superior. Arch Oftalmol B Aires 1976;51:267-71.

9. Prieto-Díaz J, Souza-Dias C. Estrabismo, $3^{\text {a }}$ edição J de Poch, La Plata, 1996, 369.

10. Souza-Dias C. Full tenectomy of superior oblique muscle close to scleral insertion for the correction of the "A" anisotropia. Proceedings of the fifth meeting, ISA-Rome 1986, E. Campos, p. 429-36.

11. Velez G. Graduated tenotomy of superior oblique by temporal approach to "A" - pattern anisotropia. Binocular Vision Qtrly 1987;2:217-20.

12. Vempalli VMR, Lee JPL. Results of superior oblique posterior tenotomy. JAAPOS 1998;2(3):147-50.

\section{Novidades na Internet!:!}

Agora no site CBO você tem disponível todas as informações na íntegra dos

Arquivos Brasileiros de Oitalmologia

http://www.cho.com.br/abo 